\title{
Correlation Study on HLA-DR and CDII7 (c-Kit) Expressions: Its Prognosis and Treatment Response in Acute Myeloid Leukemia Patients
}

This article was published in the following Dove Press journal: Pharmacogenomics and Personalized Medicine

\author{
Yomna M El-Meligui iD \\ Heba E Abd Elrhman ${ }^{2}$ \\ Ahmad Salahuddin (iD ${ }^{3}$ \\ Manal Ali Hamouda ${ }^{4}$ \\ Amira B Kassem (iD ${ }^{5}$ \\ 'Department of Clinical Pathology, \\ National Cancer Institute, Cairo \\ University, Cairo, Egypt; ${ }^{2}$ Department of \\ Clinical Pathology, Faculty of Medicine, \\ Zagazig University, Zagazig, Egypt; \\ ${ }^{3}$ Biochemistry Department, Faculty of \\ Pharmacy, Damanhour University, \\ Damanhour, Egypt; ${ }^{4}$ Clinical Pharmacy \\ Department, Faculty of Pharmacy, Ahram \\ Canadian University, Giza, Egypt; ${ }^{5}$ Clinical \\ Pharmacy and Pharmacy Practice \\ Department, Faculty of Pharmacy, \\ Damanhour University, Damanhour, \\ Egypt
}

Introduction: Acute myeloid leukemia (AML) is the most common type of acute leukemia in adults. HLA-DR and CD117 (c-Kit) are important diagnostic markers of AML. Our objective is to determine the prognostic significance of HLA-DR and CD117 expressions in newly diagnosed AML patients and determine the correlation between HLA-DR and CD117 expressions and other prognostic markers such as cytogenetic abnormalities, FLT3ITD, response to treatment, and patient's survival.

Methods: This study included 100 newly diagnosed AML patients. All patients were subjected to clinical, morphological, cytochemical, cytogenetic analysis, molecular genetic analysis to detect FLT3-ITD, and Flowcytometric detection of HLA-DR, CD117, and CD 34. Results: The results showed that HLA-DR expression was found in 75 patients $(77.3 \%)$, while CD117 expression was found in 63 patients (64.9\%). Patients with HLA-DR expression showed significantly higher mean $\mathrm{Hb}$ concentration, significantly higher platelet count, associated with AML-FAB subtypes (M0, M1, and M2), CD34 expression, and favorable cytogenetic group. M3 subtype was significantly associated with HLA-DR-ve. While patients with CD117 expression showed significantly lower platelets count. Double positive patients (HLA-DR+ve/CD117+ve) showed significant association with the intermediate cytogenetic group, while double-negative patients (HLA-DR-ve/CD117-ve) were associated with the favorable and intermediate cytogenetic group and either positive (HLA-DR+ve /CD117-ve or HLA-DR-ve/CD117+ve) associated with poor cytogenetic groups. FLT3-ITD expression had significantly worse overall survival.

Conclusion: The current study suggested that the expression of CD117 and HLA-DR may be a prognostic marker in AML, as they are associated with M0, M1, and M2 FAB subtypes; moreover, patients with combined HLA-DR and CD117 positive expression are associated with CD34 expression and intermediate cytogenetic group.

Keywords: acute myeloid leukemia, HLA-DR, CD117, CD34, FLT3-ITD, response, cytogenetic

\section{Introduction}

Acute myeloid leukemia (AML) is the most common type of acute leukemia in adults. AML is a heterogeneous group of malignant disorders characterized by the expansion of immature myeloid precursors in bone marrow (BM) and peripheral blood (PB). ${ }^{1}$ It results from acquiring genetic defects in either hematopoietic stem cells or progenitor cells, which lead to chromosomal rearrangement of multiple genes and finally malignant transformation. ${ }^{1}$ Without treatment, AML is fatal 
within a short time. Although most young patients can achieve complete remission (CR) with chemotherapy but many of them relapse. ${ }^{2}$

Therefore, the identification of new prognostic and predictive markers is of great importance. In particular, those potentially targeting individualized therapeutic options. $^{3}$ The expression of antigens on the cell surface in AML is becoming an essential research point related to prognostic effect and potential treatment implications. ${ }^{4}$

The receptor tyrosine kinase CD117 (c-Kit) is expressed on hematopoietic stem cells' surface and is essential in the survival, proliferation, and differentiation of the cells. CD117 is a $145-\mathrm{kD}$ tyrosine kinase transmembrane receptor expressed in $4 \%$ of mononuclear cells of normal bone marrow, including original stem cells, mast cells, and progenitor cells. It is expressed in around $85 \%$ of AML cases. ${ }^{5}$

Additionally, in a subset of patients, specifically those with core-binding factor (CBF) AML (inv(16)/t(16; 16) and $\mathrm{t}(8 ; 21)$, mutations in $\mathrm{c}-\mathrm{Kit}$ were known to be established. ${ }^{4}$ As there is an interest in using tyrosine kinase inhibitors with the anti-KIT activity, which are based on surface CD117 expression as a therapy in AML, we sought to correlate the association of CD117 expression with biologic and clinical data in patients with AML. ${ }^{6}$

Human leukocyte antigens Class II (HLA) present antigenic peptides to regulatory T cells. HLA Class II antigens' role in producing the immune response has stimulated interest in determining whether tumor cellexpressed HLA Class II antigens affect the disease's clinical course. ${ }^{7}$ HLA-DR has been utilized to distinguish a particular type of AML, such as acute promyelocytic leukemia (APL) or AML-M3 from other AML subtypes (M0, M1, M2, M4, M5, M6, and M7) as categorized based on the 1976 French-American-British (FAB) Classification. ${ }^{4}$

Absent HLA-DR, missing or dim CD34, and a robust CD117 expression were representative of classical APL. However, the absence of CD34 or HLA-DR was noted in a fair percentage of non-APL cases as well. ${ }^{8}$

Approximately $30 \%$ of all AML cases express mutations of the FMS-like tyrosine kinase 3 (FLT3) gene. The internal tandem duplication (ITD) represents the most common type of FLT3 mutation. FLT3-ITD is a mutation that presents with a poor prognosis in AML patients. Earlier identification of FLT3-ITD might enable targeted treatment to help patients achieve more prolonged and more durable remissions. ${ }^{9}$ Cytogenetic analysis can detect any structural or numerical aberration, novel, and uncharacterized abnormalities. WHO classification has focused on the importance of cytogenetic abnormalities in the subtyping of leukemias. ${ }^{10}$

The objective of this study is to determine the prognostic significance of HLA-DR and CD117 expressions in newly diagnosed AML patients and determine the correlation between HLA-DR and CD117 expressions and other prognostic markers as cytogenetic abnormalities, FLT3ITD, response to treatment, and patient's survival.

\section{Patients and Methods}

This study was conducted in the National Cancer Institute (NCI), Cairo University, Egypt, and included 100 patients with de novo AML referred to Medical Oncology Department between March 2018 and February 2020. Eligible patients were those age 18 years at least, with a confirmed diagnosis of AML, and had no contraindications to induction of chemotherapy. All patients were subjected to clinical, morphological, cytochemical, cytogenetic analysis, molecular genetic analysis to detect FLT3-ITD, and Flowcytometric detection of HLA-DR, CD117, and CD 34 to establish the diagnosis and to assess risk stratification before starting the chemotherapy. Plain chest X-ray or CT chest, abdominal and pelvic ultrasonography, as well as echocardiography, were routinely done before induction treatment. The study was approved by the Institutional Review Board (IRB) of the NCI, Cairo University, and all procedures were following the Declaration of Helsinki.

\section{Therapeutic Regimens}

A uniform treatment protocol was followed for all patients. This involved induction with a $3+7$ regimen (daunorubicin $45 \mathrm{mg} / \mathrm{m}^{2}$ for three consecutive days and cytosine arabinoside $100 \mathrm{mg} / \mathrm{m}^{2}$ for another seven days) followed by two cycles of a $5+2$ protocol after remission (daunorubicin $45 \mathrm{mg} / \mathrm{m}^{2}$ for two days, plus cytosine arabinoside $100 \mathrm{mg} / \mathrm{m}^{2}$ for another five days).

Patients who were not in complete remission (CR) morphologically after the first chemotherapy session received cytosine arabinoside $500 \mathrm{mg} / \mathrm{m}^{2}$ by slow intravenous push twice a day for seven days and Mitoxantrone $12 \mathrm{mg} / \mathrm{m}^{2}$ daily for three days. ${ }^{11}$

\section{Efficacy and Follow-Up}

Efficacy of the chemotherapy was evaluated using the response criteria for leukemia provided by International 
Working Group (IWG) criteria for AML as follows: complete remission (CR): the recovery of normal bone marrow aspirates and blood counts (blast cells less than 5\%, platelet count $\geq 100 \times 10^{9} / \mathrm{L}$, absolute neutrophilic count (ANC) $\geq 1.0 \times 10^{9} / \mathrm{L}$ with no evidence of extramedullary leukemia); primary resistance (PR): (blast cells cellular marrow with $>5 \%$ blast cells); early relapse (ER): (relapse occurred within six months after remission). Both PR and ER were accounted for all the early failures. ${ }^{12}$

Overall survival (OS) was measured from the diagnosis date to the death date or last follow-up date in February 2020. Disease-free survival (DFS) was measured from CR date until the date of relapse or death.

\section{Flowcytometric Analysis of HLA-DR, CDII7}

$\mathrm{BM}$ and PB/EDTA samples were obtained at diagnosis and analyzed within 24 hours. Complete routine panel of monoclonal antibodies for AML patients used: CD2, CD3, CD4, CD5, CD7, CD8, CD10, CD11c, CD13, CD14, CD19, CD20, CD22, CD33, CD34, CD45, CD64, cytoplasmic MPO and TdT. Briefly, $100 \mathrm{ul}$ of the sample with an adjusted cell count of approximately $1 \times 10^{6}$ cells / tube were incubated with monoclonal according to the manufacturer's recommendations in the dark for $30 \mathrm{~min}$ utes at room temperature, then lysed and washed with phosphate buffer saline (PBS) to get rid of excess antibodies. Cells were resuspended in PBS.

Mouse anti-human HLA-DR and CD117 monoclonal antibodies (catalog no.: 347363, and 341126 respectively) were purchased from (BD Biosciences, CA, USA) were added at diagnosis. Isotype/negative control (IgG1) stained with PE or FITC was used.

According to the manufacturers' protocol, samples were run on a multicolor flow cytometer (FACS Canto; BD Biosciences, San Jose, CA, USA). The analysis was performed using the FACS DIVA 6.1.3 software (BD Biosciences).

Gating strategy: Initial gating was done using typical forward- scatter (FSC) versus side scatter (SSC) on the blast area, then from cd45 (FITC conjugated) versus side scatter, so gating strategy was based on CD45.

Blast area evaluated for expression of HLA-DR and CD117 using quadrant plot where CD117 (PE-conjugated) was represented on the Y-axis, and HLA-DR (FITC conjugated) was represented on $\mathrm{X}$-axis. The co-expression area was manifested in the upper right quadrant, whereas cells negative for both markers were located in the lowerleft quadrant, cells positive for CD117 only were located on the upper left quadrant, and cells positive for HLA-DR only were located on the lower right quadrant. Single histograms were used for each marker versus side scatter.

In total, 10,000 events were required, and the percent expressions of HLA-DR and CD117 on gated myeloblasts were recorded. Markers' positivity was considered if $\geq 20 \%{ }^{13}$

Patients were divided into positive and negative groups, then positive HLA-DR and positive CD117 groups were taken together, negative HLA-DR and CD117 groups were taken together, and either positive was taken [Figure 1].

\section{Analysis of FLT3 Gene Mutations}

According to the manufacturer's protocol, high molecular weight DNA was extracted from BM/EDTA samples using QIAamp DNA Blood Mini Kit (QIAGEN). PCR amplification was carried out using FLT3-ITD master mix contained dNTPs, the forward (5'-CAATTTAGGTATGAAA GCC-3 ') and reverse (5'-GTACCTTTCAGCATTTTGAC $-3^{\prime}$ ) primers (Invivoscribe Technologies, Inc., USA). Positive and negative control DNAs (Invivoscribe Technologies, Inc., USA), AmpliTaq DNA polymerase (Applied Biosystems, Life Technologies, USA), and 100 bp DNA ladder (Invitrogen, Life Technologies, USA) were used.

In brief, 1ul DNA was amplified in a volume of $25 \mu \mathrm{L}$ containing $50 \mathrm{mM} \mathrm{KCl,} 10 \mathrm{mM}$ Tris- $\mathrm{HCl}, \mathrm{PH} 8.3,1.5 \mathrm{Mm}$ $\mathrm{MgCl} 2,200 \mathrm{mM}$ dNTPs, $0.5 \mathrm{uM}$ of each primer and $1 \mathrm{U}$ Taq DNA polymerase (QIAGEN). The PCR consisted of an initial incubation step at $94{ }^{\circ} \mathrm{C}$ for 150 seconds followed by 35 cycles at $94{ }^{\circ} \mathrm{C}$ for 30 seconds, $57{ }^{\circ} \mathrm{C}$ for 60 seconds, and $72{ }^{\circ} \mathrm{C}$ for 120 seconds, and a final elongation step at $94{ }^{\circ} \mathrm{C}$ for 30 seconds and $60{ }^{\circ} \mathrm{C}$ for 10 minutes. The PCR product was analyzed on standard 3\% agarose gel stained with ethidium bromide. A fragment of 328 base pairs (bp) was produced from wild-type (WT) alleles. All patient with an additional higher molecular weight band was considered to be FLT3/ITD+.

\section{Statistical Methods}

Statistical analysis was done using IBM SPSS ${ }^{\circledR}$ Statistics version 22 (IBM ${ }^{\circledR}$ Corp., Armonk, NY, USA). Numerical data were described as a mean and median. Also, qualitative data described as frequency and percentage. Pearson's Chi-square test or Fisher's exact test was employed to 


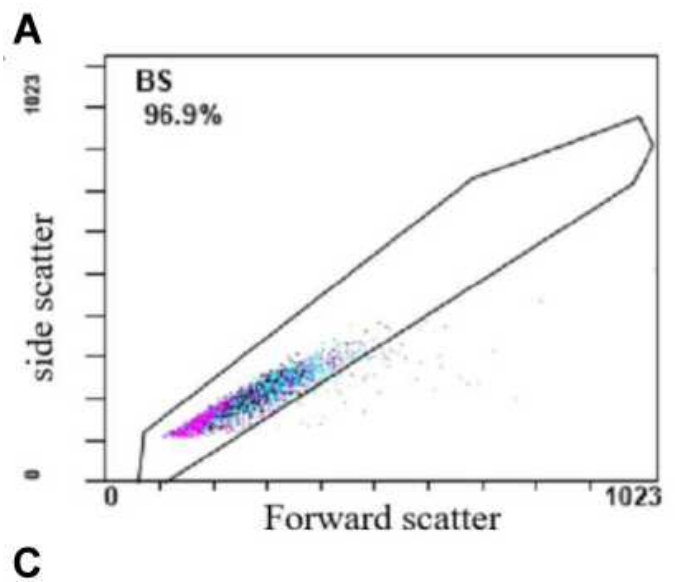

\section{B}
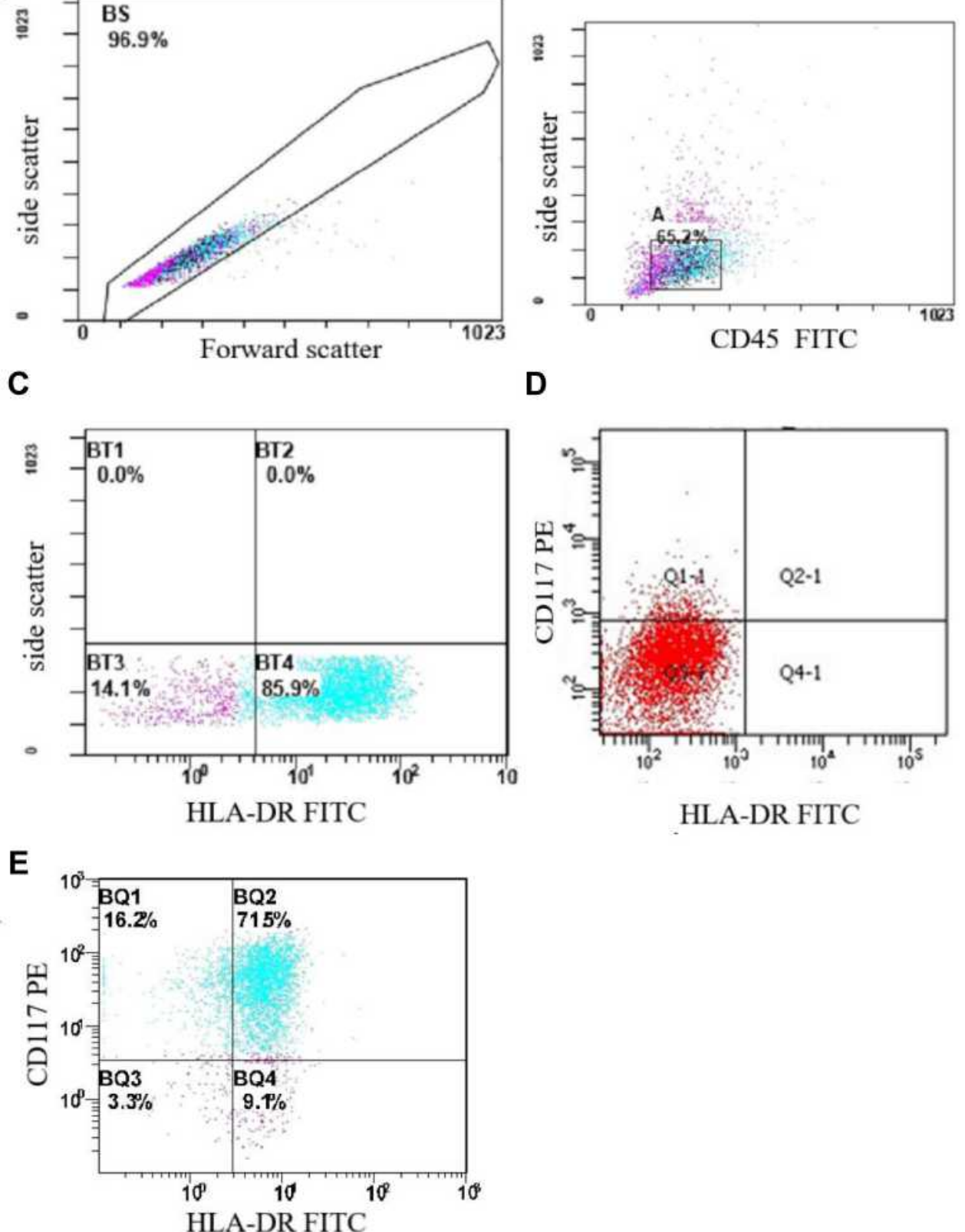

Figure I (A) Illustrates forward scatter versus side scatter on blast area. (B) Illustrates CD45 versus side scatter on blast area. (C) Flow histogram showing HLA-DR positive HLA-DR on myeloblasts. (D) Flow histogram showing negative CDII7 and negative HLA-DR on myeloblasts. (E) Flow histogram showing positive CDII7 and positive HLA-DR on myeloblasts.

analyze the relationship between qualitative variables. Quantitative data were tested for normality using the Kolmogorov-Smirnov test and Shapiro-Wilk test. Comparison between two groups was made using either Student's $t$-test for normally distributed quantitative data while Mann-Whitney test (non-parametric $t$-test) was used for not normally distributed quantitative data. Comparison between the three groups was made using the KruskalWallis test (non-parametric ANOVA). Survival analysis was conducted using the Kaplan-Meier equation, and the Log rank test was used to compare two survival curves. All tests were two-tailed. A p-value $<0.05$ was considered significant.

\section{Results}

\section{Patients' Characteristics}

One hundred de novo AML patients were enrolled in the study. The clinicopathological characteristics of the patients 
Table I Clinico-Pathological Characteristics of 100 Adult Acute Myeloid Leukemia Patients

\begin{tabular}{|c|c|}
\hline Parameter & Finding \\
\hline \multirow[t]{2}{*}{ Age: Years* } & $40.5 \pm 14.3$ \\
\hline & $40.5(18-70)$ \\
\hline \multicolumn{2}{|l|}{ Gender: $(n=100)$} \\
\hline Males & 48 (48\%) \\
\hline Females & $52(52 \%)$ \\
\hline \multirow[t]{2}{*}{ Hemoglobin: gm/d* } & $7.9 \pm 1.8$ \\
\hline & $7.8(3.1-12.3)$ \\
\hline \multirow[t]{2}{*}{ TLC: $\times 10^{9} / L^{*}$} & $51.9 \pm 68.5$ \\
\hline & $26.1(I-345)$ \\
\hline \multirow[t]{2}{*}{ Platelets: $\times 10^{9} / L^{*}$} & $58 \pm 72.9$ \\
\hline & $36(1-436)$ \\
\hline \multicolumn{2}{|l|}{ Blast: \%* } \\
\hline Peripheral blood & $51.8 \pm 26.5$ \\
\hline Bone marrow & $62.6 \pm 20.3$ \\
\hline Lymph node enlargement: No (\%) & $21 / 97(21.6 \%)$ \\
\hline Hepatomegaly: No (\%) & $42 / 97(43.3 \%)$ \\
\hline Splenomegaly: No (\%) & $36 / 97(37.1 \%)$ \\
\hline Morphology (FAB types): & $n=100$ \\
\hline Mo & 1 \\
\hline MI & 22 \\
\hline M2 & 39 \\
\hline M3 & 8 \\
\hline M4 & 23 \\
\hline M5 & 5 \\
\hline M7 & 2 \\
\hline Cytogenetic analysis $(n=60)$ & No (\%) \\
\hline Favorable cytogenetic: & $17(28.3 \%)$ \\
\hline Intermediate cytogenetic: & $21(35 \%)$ \\
\hline Poor cytogenetic: & $22(36.7 \%)$ \\
\hline Unfavorable genetics: FLT3-ITD: No (\%) & $14 / 82(17.1 \%)$ \\
\hline CD34 expression: No (\%) & $52 / 97(53.6 \%)$ \\
\hline CDII7 expression: No (\%) & $63 / 97(64.9 \%)$ \\
\hline HLA-DR expression: No (\%) & 75/97 (77.3\%) \\
\hline CDII7. HLA-DR expressions ( $n=97$ ) & No (\%) \\
\hline Double-positive & $50(51.5 \%)$ \\
\hline Double-negative & $9(9.3 \%)$ \\
\hline Single +ve & $38(39.2 \%)$ \\
\hline Response at day $28(n=93)$ & No (\%) \\
\hline $\mathrm{CR}^{* *}$ & $47(50.5 \%)$ \\
\hline Delayed CR & $10(10.8 \%)$ \\
\hline Early death & $28(30.1)$ \\
\hline
\end{tabular}

(Continued)
Table I (Continued).

\begin{tabular}{|l|c|}
\hline Parameter & Finding \\
\hline Resistant*** & $8(8.6 \%)$ \\
\hline Median survival time (months) ${ }^{* * *}$ & 4.11 \\
\hline
\end{tabular}

Notes: *Mean \pm SD, Median (range). $* * C R$ percentage was calculated who achieved complete response. ${ }^{* * *}$ Survival data were available for 93 patients.

are shown in [Table 1]. AML M2 was the most frequent FAB subtype representing $39 \%$ of the patients, followed by M4 and M1, representing $23 \%$ and $22 \%$, respectively.

Cytogenetic analysis of these cases showed normal karyotype in 19 (31.7\%) cases and abnormal karyotype in $41(68.3 \%)$ cases. There were three groups of cytogenetic findings in AML: favorable risk, intermediate-risk, and unfavorable risk. There were $17(28.3 \%)$ cases in the favorable risk group, and cytogenetic abnormality includes cases with the $t(8 ; 21)$, inv(16), and the $t(15 ; 17)$. There were $21(35 \%)$ cases in the intermediate-risk group, including trisomy 3 , trisomy 8 , monosomy 22 , and normal karyotype. The unfavorable group includes 22 (36.7\%) cases with other karyotypes [Table 2].

Also, unfavorable genetic mutation like FLT3-ITD was detected in 14 AML patients (17.1\%). Ninety-three patients received full induction chemotherapy with a $3+7$ regimen. On day 28,47 patients $(50.5 \%)$ achieved CR, 28 $(30.1 \%)$ died during the induction period, while $8(8.6 \%)$ patients were refractory to treatment.

\section{Expression of HLA-DR and CDII7 in Newly Diagnosed AML Patients}

Out of 97 patients assessed, HLA-DR expression was found in 75 patients (77.3\%), while CD117 expression was found in 63 patients (64.9\%). Patients with HLA-DR expression showed significant higher mean hemoglobin concentration ( $8.1 \pm 1.9$ vs $7.2 \pm 1.4, \mathrm{p}=0.04$ ), significantly higher platelets count $(66.1 \pm 79.3$ vs $30.1 \pm 32.4, \mathrm{p}=0.012)$, and nonsignificant higher total leukocyte count (TLC) (56.7 772.1 vs $35 \pm 54.6, \mathrm{p}=0.08$ ), but there was a significant association between HLA-DR expression and higher TLC more than $11 \mathrm{x}$ $10^{9} / \mathrm{L}$ ( $72 \%$ vs $45.5, \mathrm{p}=0.002$ ). Also, HLA-DR expression associated with lower peripheral blood blast percentage (50.8 \pm 27.8 vs $54.2 \pm 22.6, \mathrm{p}=0.59)$ and lower bone marrow blast percentage ( $61 \pm 20$ vs $67.4 \pm 21.1, \mathrm{p}=0.12)$. Significant associations were found between AML-FAB subtypes (M0, M1, and M2) and positive HLA-DR expression $(p<0.001)$. 
Table 2 Cytogenetic Analysis Findings in 60 Adult Acute Myeloid Leukemia Patients

\begin{tabular}{|c|c|c|}
\hline Cytogenetic Analysis & Frequency & Percent \\
\hline $\mathrm{t}(15: 17)$ & 8 & 13.3 \\
\hline $\mathrm{t}(8: 21)$ & 7 & 11.7 \\
\hline$t(8: 2 I)$, tri 8 & 1 & 1.7 \\
\hline invl6 & 1 & 1.7 \\
\hline NK (normal karyotype) & 19 & 31.7 \\
\hline tri3 & 1 & 1.7 \\
\hline tri8, mono22 & 1 & 1.7 \\
\hline monol 5, 20, 22 & 1 & 1.7 \\
\hline monol6, 4q+ & 1 & 1.7 \\
\hline monol2, 21, tril9 & 1 & 1.7 \\
\hline mono5, 13, 17, 22 & 1 & 1.7 \\
\hline monol7, 2I, invlq & 1 & 1.7 \\
\hline$t(6: 17),+17 p$ & 1 & 1.7 \\
\hline 47, xy, tril9 & 1 & 1.7 \\
\hline$t(9: 22)$ & 7 & 11.7 \\
\hline$t(9: 22)$, tri8 & 1 & 1.7 \\
\hline $\mathrm{t}(9: 22)$, mono7 & I & 1.7 \\
\hline trill & 1 & 1.7 \\
\hline tri2l & 2 & 3.3 \\
\hline tri8, 13,17, mono22 & 1 & 1.7 \\
\hline tri8, mono2I & 1 & I.7 \\
\hline Tri2 & I & 1.7 \\
\hline Total & 60 & 100.0 \\
\hline
\end{tabular}

Positive HLA-DR expression was significantly associated with the intermediate cytogenetic group (41.9\%), while negative HLA-DR expression associated with the favorable cytogenetic group $(56.2 \%)(\mathrm{p}=0.017)$ and positive HLA-DR expression showed significant association with CD34 expression $(\mathrm{p}=0.005)$.

Patients with CD117 expression showed significantly lower platelets count $(53.6 \pm 77.7$ vs $66.1 \pm 63.2, p=0.02)$ and non-significantly lower TLC (45.5 56.9 vs $63.3 \pm 86.6$, $\mathrm{p}=0.91)$, higher peripheral and bone marrow blood blast percentage (54 \pm 27.4 vs $47 \pm 25.1, \mathrm{p}=0.22)$ and $(62.7 \pm 21$ vs $62.1 \pm 19.3, \mathrm{p}=0.74)$ respectively. Significant associations were found between CD117 expression and lymph node enlargement ( $\mathrm{p}=0.001)$. In contrast to HLA-DR, no significant association with either favorable, intermediate, or unfavorable cytogenetics could be demonstrated, and no significant association with AML-FAB subtypes and CD34 expression (Table 3).

\section{HLA-DR and CDII7 Expression and Complete Remission Rates}

Patients with HLA-DR expression showed non-significant worse CR rates at day 28 than those without HLA-DR expression (50\% and $52.4 \%$, respectively). Also, patients with HLA-DR expression showed a lower incidence of early death during induction than those without expression (27.7\% versus $38.1 \%$ ). In contrast, patients with CD117 expression showed better CR rates at day 28 than patients without $(56.7 \%$ versus $39.4 \%)$. Patients with CD117 expression showed a lower incidence of early death during induction than those without CD117 expression (23.3\% versus $42.4 \%$ ) (Table 3 ).

\section{Correlation of Survival Rates with HLA-DR and CDII7 Expression}

After a median follow-up period of 4.1 months with a range (0.03 to 24.87 months), patients with HLA-DR expression showed increased median Overall survival (OS) compared to patients without HLA-DR expression (5.4 versus 2.7 months, $\mathrm{p}=0.7$ ). CD117 expression showed longer median OS than patients without expression (5.1 versus 4.4 months; $p=0.9$ ) [Figure 2].

\section{Combined Expression of HLA-DR and CDII7 in AML Patients}

According to the immunophenotypic expression of HLA-DR and CD117 on 97 AML patients, patients were divided into 3 groups: HLA-DR +/CD117+ (Double positive) ( $\mathrm{n}=50,51.5 \%)$, HLA-DR-/CD117(Double negative) $(\mathrm{n}=9,9.3 \%)$ and HLA-DR +/CD117or HLA-DR -/CD117+ (Either positive) $(\mathrm{n}=38,39.2 \%)$ (Table 4).

No statistical significance was found between all groups and age $(\mathrm{p}=0.4)$, gender $(\mathrm{p}=0.3)$, mean $\mathrm{Hb}$ concentration $(\mathrm{p}=0.3)$, platelets count $(\mathrm{p}=0.6)$, TLC $(\mathrm{p}=0.08)$, peripheral blood blast percentage $(\mathrm{p}=0.6)$, bone marrow blast percentage $(\mathrm{p}=0.4)$. Significant associations were found in AMLFAB subtypes (M0, M1, and M2) between double negative and double-positive patients (HLA-DR $+/ \mathrm{CD} 117+$ ) $(\mathrm{p}=0.037)$ double-positive patients showed significant 
Table 3 Clinical and Hematological Findings of Acute Myeloid Leukemia Patients According to HLA-DR and CDII7 Expression

\begin{tabular}{|c|c|c|c|c|c|c|}
\hline Parameter & $\begin{array}{l}\text { HLA-DR -ve } \\
\qquad(n=22)\end{array}$ & $\begin{array}{l}\text { HLA-DR +ve } \\
\quad(n=75)\end{array}$ & p-value & $\begin{array}{l}\text { CDII7 - ve } \\
(n=34)\end{array}$ & $\begin{array}{l}\text { CDII } 7+v e \\
(n=63)\end{array}$ & p-value \\
\hline Age: Years* & $42.9 \pm 14.7$ & $39.2 \pm 13.8$ & NS & $41.6 \pm 13.2$ & $39.2 \pm 14.5$ & NS \\
\hline Gender: Males: Female & $12: 10(1.2: 1)$ & $35: 40(0.9-1)$ & NS & $16: 18(0.9: 1)$ & $31: 32(0.96: 1)$ & NS \\
\hline Hemoglobin $(\mathrm{gm} / \mathrm{dl}) *$ & $7.2 \pm 1.4$ & $8.1 \pm 1.9$ & 0.04 & $7.9 \pm 1.8$ & $7.9 \pm 1.8$ & NS \\
\hline TLC: $\times 10^{9} / \mathrm{L} *$ & $35 \pm 54.6$ & $56.7 \pm 72.1$ & 0.08 & $63.3 \pm 86.6$ & $45.5 \pm 56.9$ & NS \\
\hline TLC > $11 \times 10^{9} / \mathrm{L}$ No (\%) & $10 / 22$ (45.5\%) & $54 / 75(72 \%)$ & 0.02 & $23 / 34(67.6 \%)$ & $41 / 63(65.1 \%)$ & NS \\
\hline Platelets: $\times 10^{9} / \mathrm{L} *$ & $30.1 \pm 32.4$ & $66.1 \pm 79.3$ & 0.012 & $66.1 \pm 63.2$ & $53.6 \pm 77.7$ & 0.02 \\
\hline Blasts: \%*: & & & & & & \multirow{4}{*}{$\begin{array}{c}\text { NS } \\
0.001\end{array}$} \\
\hline Peripheral blood & $54.2 \pm 22.6$ & $50.8 \pm 27.8$ & NS & $47.1 \pm 25.1$ & $54 \pm 27.4$ & \\
\hline Bone marrow & $67.4 \pm 21.1$ & $61 \pm 20$ & NS & $62.1 \pm 19.3$ & $62.7 \pm 21$ & \\
\hline Lymph node enlargement, No (\%) & $5 / 22(22.7 \%)$ & $16 / 75(21.3 \%)$ & NS & $1 / 34(2.9 \%)$ & $20 / 63(31.7 \%)$ & \\
\hline Hepatomegaly: No (\%) & $8 / 22(36.4 \%)$ & $34 / 75(45.3 \%)$ & NS & 13/34 (38.2\%) & $29 / 63(46 \%)$ & NS \\
\hline Splenomegaly No (\%) & $6 / 22(27.3 \%)$ & $30 / 75(40 \%)$ & NS & 13/34 (38.2\%) & $23 / 63(36.5 \%)$ & NS \\
\hline FAB subtypes: & & & $<0.001$ & $17 / 34(50 \%)$ & $43 / 63(68.2 \%)$ & NS \\
\hline M0, MI, M2 & $12 / 22(54.6 \%)$ & $48 / 75$ (64\%) & & & & \\
\hline M3 & $7 / 22(31.8 \%)$ & $\mathrm{I} / 75(\mathrm{I} .3 \%)$ & & $3 / 34(8.8 \%)$ & $5 / 63(8 \%)$ & \\
\hline M4, M5, M7 & $3 / 22(13.6 \%)$ & $23 / 75(30.7 \%)$ & & I4/34 (4I. $2 \%)$ & $15 / 63(23.8 \%)$ & \\
\hline CD34 expression: No (\%) & $6 / 22(27.3 \%)$ & $46 / 75(61.3 \%)$ & 0.005 & $\mid 4 / 34(4 \mid .2 \%)$ & $38 / 63(60.3 \%)$ & 0.07 \\
\hline CDII7 expression: No (\%) & $13 / 22(59.1 \%)$ & $50 / 75(66.7 \%)$ & NS & - & - & - \\
\hline HLA-DR expression: No (\%) & - & - & - & $25 / 34(73.5 \%)$ & $50 / 63(79.4 \%)$ & NS \\
\hline Cytogenetic analysis: & & & 0.017 & $5 / 23(21.8 \%)$ & $12 / 36(33.3 \%)$ & NS \\
\hline Favorable cytogenetics & $9 / 16$ (56.2\%) & $8 / 43(18.6 \%)$ & & & & \\
\hline Intermediate cytogenetics & $3 / 16$ (18.8\%) & I8/43 (4I.9\%) & & $7 / 23(30.4 \%)$ & $14 / 36(39 \%)$ & \\
\hline Poor cytogenetics & $4 / 16(25 \%)$ & I7/43 (39.5\%) & & I I/23 (47.8\%) & $10 / 36(27.7 \%)$ & \\
\hline FLT3-ITD & $5 / 17$ (29.4\%) & $9 / 65$ (13.8\%) & NS & $3 / 30(10 \%)$ & $1 \mathrm{I} / 52$ (2I.1\%) & NS \\
\hline $\begin{array}{l}\text { Response to treatment at D28 } \\
(n=93) \text { : }\end{array}$ & $N=21$ & $N=72$ & NS & $N=33$ & $N=60$ & 0.088 \\
\hline$C R(n=47) * *$ & II (52.7\%) & $36(50 \%)$ & & 13 (39.4\%) & 34 (56.7\%) & \\
\hline Delayed CR $(n=10)$ & I (4.6\%) & $9(12.5 \%)$ & & $5(15.2 \%)$ & 5 (8.3\%) & \\
\hline Resistant $(n=8)$ & I (4.6\%) & 7 (9.6\%) & & I (3\%) & 7 (II.7\%) & \\
\hline Early death $(n=28)$ & $8(38.1 \%)$ & 20 (27.7\%) & & 14 (42.4\%) & 14 (23.3\%) & \\
\hline $\begin{array}{l}\text { Median survival time/DFS (months) } \\
(n=57)\end{array}$ & 12.8 & 11.1 & NS & $* * *$ & 12.1 & NS \\
\hline $\begin{array}{l}\text { Median survival time/OS (months) } \\
(n=93)\end{array}$ & 2.9 & 5.4 & NS & 4.4 & 5.1 & NS \\
\hline
\end{tabular}

Notes: *Mean $\pm \mathrm{SD}$. ${ }^{*} \mathrm{CR}$ percentage was calculated who achieved a complete response. ${ }^{* *}$ No median because more than half of the patients were free till the end of the study.

association with the intermediate cytogenetic group. In contrast, double-negative patients (HLA-DR-/CD117-) were associated with the favorable and intermediate cytogenetic group, and either positive (HLA-DR +/CD117- or HLA-DR
$-/ \mathrm{CD} 117+)$ were associated with poor cytogenetic groups $(\mathrm{p}=0.04)$. Double-positive patients (HLA-DR $+/ \mathrm{CD} 117+$ ) also showed significant associations with $\mathrm{CD} 34$ expression $(\mathrm{p}=0.042)$ (Table 4). 

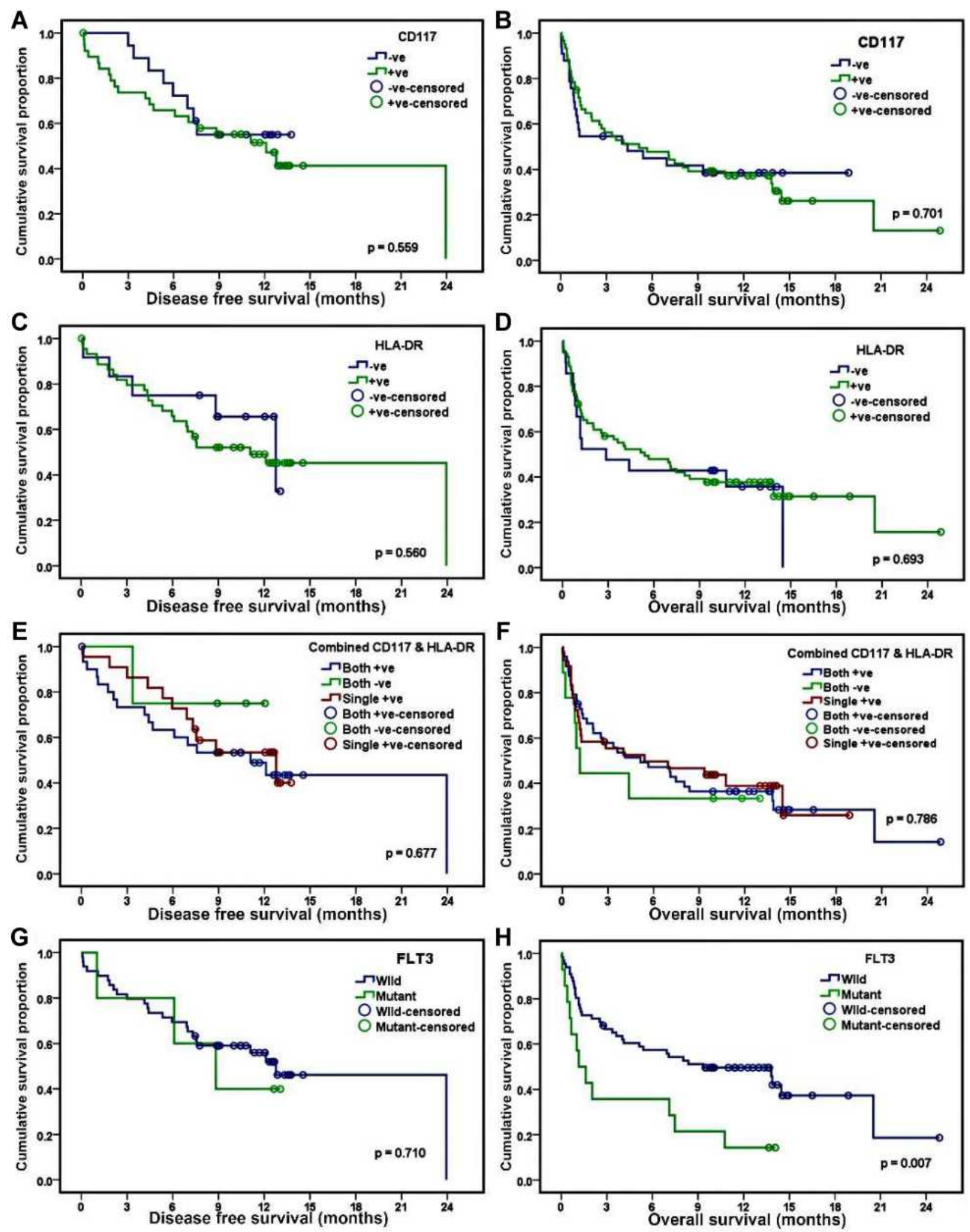

Figure 2 (A, C, E, G) Kaplan-Meier curves with log-rank statistics describe disease-free survival according to CD I I7, HLA-DR, Combined CD I I7 and HLA-DR, and FIt3 expression level. (B, D, F, H) Kaplan-Meier curves with log-rank statistics describe overall survival according to CDI I7, HLA-DR, Combined CDII7 and HLA-DR, and FIt3 expression level. 
Table 4 Clinical and Hematological Findings of Acute Myeloid Leukemia Patients According to Combined HLA-DR and CDII7 Expression

\begin{tabular}{|c|c|c|c|c|}
\hline \multirow[t]{2}{*}{ Parameter } & \multicolumn{3}{|c|}{ CDIIT/ HLA-DR } & \\
\hline & Double +ve $(n=50)$ & Double -ve (n=9) & Single +ve $(n=38)$ & \\
\hline Age: Years* & $38.2 \pm 14.2$ & $42.4 \pm 14.3$ & $41.9 \pm 13.8$ & NS \\
\hline Gender: Male: Female & $22: 28(0.8: 1)$ & $3: 6(1: 2)$ & $22: 16(1.4: 1)$ & NS \\
\hline Hemoglobin $(\mathrm{gm} / \mathrm{dl}) *$ & $8.1 \pm 1.9$ & $7.1 \pm 1.3$ & $7.9 \pm 1.8$ & NS \\
\hline TLC: $\times 10^{9} / \mathrm{L} *$ & $54.2 \pm 54.7$ & $18.2 \pm 23.2$ & $68.3 \pm 87.2$ & NS \\
\hline TLC $>11 \times 10^{9} / \mathrm{L}$ No $(\%)$ & $35 / 50(70 \%)$ & $4 / 9(44.4 \%)$ & $25 / 38(65.8 \%)$ & NS \\
\hline Platelets: $\times 10^{9} / \mathrm{L} *$ & $59.8 \pm 83.9$ & $31.2 \pm 16.1$ & $61.8 \pm 64.9$ & NS \\
\hline Blasts: \%* & & & & \\
\hline Peripheral blood & $53.7 \pm 28$ & $53 \pm 18.4$ & $48.4 \pm 26.8$ & NS \\
\hline Bone marrow & $62.3 \pm 20.2$ & $71.8 \pm 15.5$ & $60.6 \pm 21.2$ & NS \\
\hline Lymph node enlargement, No (\%) & $15 / 50(30 \%)$ & $0 / 9(0 \%)$ & $6 / 38(15.8 \%)$ & 0.07 \\
\hline Hepatomegaly: No (\%) & $24 / 50(36.4 \%)$ & $3 / 9(30 \%)$ & $15 / 38(39.5 \%)$ & NS \\
\hline Splenomegaly No (\%) & $19 / 50(38 \%)$ & $2 / 9(22.2 \%)$ & I5/38 (39.5\%) & NS \\
\hline FAB subtypes: & & & & 0.037 \\
\hline M0, MI, M2 & $34 / 50(68 \%)$ & $3 / 9(30 \%)$ & $23 / 38(64 \%)$ & \\
\hline M3 & $1 / 50(2 \%)$ & $3 / 9(30 \%)$ & $4 / 38$ (1.3\%) & \\
\hline M4, M5, M7 & $15 / 50(30 \%)$ & $3 / 9(30 \%)$ & I I/38 (30.7\%) & \\
\hline CD34 expression: No (\%) & $34 / 50(68 \%)$ & $2 / 9(22.2 \%)$ & $16 / 38(42.1 \%)$ & 0.008 \\
\hline Cytogenetic analysis: & & & & 0.04 \\
\hline Favorable cytogenetics & $6 / 27(22.2 \%)$ & $3 / 7(42.9 \%)$ & $8 / 25(32 \%)$ & \\
\hline Intermediate cytogenetics & |4/27 (5I.9\%) & $3 / 7(42.9 \%)$ & $4 / 25(16 \%)$ & \\
\hline Poor cytogenetics & $7 / 27(25.9 \%)$ & $\mathrm{I} / 7$ (14.2\%) & $13 / 25(52 \%)$ & \\
\hline FLT3-ITD & $7 / 42(16.7 \%)$ & $\mathrm{I} / 7$ (I4.3\%) & $6 / 33(18.2 \%)$ & NS \\
\hline Response to treatment at D28 $(n=93)$ : & & & & NS \\
\hline$C R(n=47) * *$ & $27 / 48(56.3 \%)$ & $4 / 9(44.4 \%)$ & $16 / 36(44.4 \%)$ & \\
\hline Delayed CR $(n=10)$ & $4 / 48(8.3 \%)$ & $0 / 9(0 \%)$ & $6 / 36(16.7 \%)$ & \\
\hline Resistant $(n=28)$ & I I/48 (22.9\%) & $5 / 9(55.6 \%)$ & $12 / 36(33.3 \%)$ & \\
\hline Early death $(n=8)$ & $6 / 48$ (12.5\%) & $0 / 9(0 \%)$ & $2 / 36(5.6 \%)$ & \\
\hline Median survival time/DFS (months) $(n=57)$ & II.I & $* * *$ & 12.8 & NS \\
\hline Median survival time/OS (months) $(n=93)$ & 5.1 & 1.2 & 5.4 & NS \\
\hline
\end{tabular}

Notes: *Mean \pm SD. $* * C R$ percentage was calculated who achieved a complete response. ${ }^{* * *}$ No median because more than half of the patients were free till the end of the study.

Patients with a combined expression of HLA-DR and CD117 showed non-significant better CR rates at day 28 compared to those with double negative and either positive groups $(56.3 \%, 44.4 \%$, and $44.4 \%$ respectively) $(\mathrm{p}=0.3)$. Survival rates showed that the double negative group had superior median DFS (as more than half of patients were free until the end of the study) compared with double-positive groups and either single positive groups (11.1 months and 12.8 months, respectively). Also, the double negative group had a superior median overall survival (1.2) months in the double negative group, which is worse than double-positive and either single positive groups (5.1 months and 5.4 months respectively) (Table 4) [Figure 2]. 
Table 5 Disease-Free Survival and Overall Survival and Its Relation to Prognostic Factors in AML Patients

\begin{tabular}{|c|c|c|c|c|c|c|c|c|c|c|}
\hline & \multicolumn{5}{|c|}{ Disease-Free Survival } & \multicolumn{5}{|c|}{ Overall Survival } \\
\hline & No & $\begin{array}{l}\text { No of } \\
\text { Events }\end{array}$ & $\begin{array}{l}\text { DFS Rate } \\
\text { (\%) at } 9 \\
\text { Months }\end{array}$ & $\begin{array}{l}\text { Median } \\
\text { Survival } \\
\text { (Months) }\end{array}$ & p-value & No & $\begin{array}{l}\text { No of } \\
\text { Events }\end{array}$ & $\begin{array}{l}\text { OS Rate } \\
\text { (\%) at } 6 \\
\text { Months }\end{array}$ & $\begin{array}{c}\text { Median } \\
\text { Survival } \\
\text { (Months) }\end{array}$ & p-value \\
\hline AML & 57 & 29 & 91.1 & 12.1 & & 93 & 61 & 93.5 & 5.1 & \\
\hline $\begin{array}{l}\text { CDII7 } \\
\text {-ve } \\
+\mathrm{ve}\end{array}$ & $\begin{array}{l}18 \\
39\end{array}$ & $\begin{array}{c}8 \\
21\end{array}$ & $\begin{array}{c}55 \\
86.8\end{array}$ & $\begin{array}{l}12.1 \\
12.1\end{array}$ & NS & $\begin{array}{l}33 \\
60\end{array}$ & $\begin{array}{l}20 \\
4 I\end{array}$ & $\begin{array}{c}87.9 \\
90\end{array}$ & $\begin{array}{l}4.4 \\
5.1\end{array}$ & NS \\
\hline $\begin{array}{l}\text { HLA-DR } \\
\text {-ve } \\
+ \text { ve }\end{array}$ & $\begin{array}{l}12 \\
45\end{array}$ & $\begin{array}{c}5 \\
24\end{array}$ & $\begin{array}{l}65.6 \\
81.8\end{array}$ & $\begin{array}{l}12.8 \\
11.1\end{array}$ & NS & $\begin{array}{l}21 \\
72\end{array}$ & $\begin{array}{l}14 \\
47\end{array}$ & $\begin{array}{l}71.4 \\
93.1\end{array}$ & $\begin{array}{l}2.9 \\
4.5\end{array}$ & NS \\
\hline $\begin{array}{l}\text { CDII7/HLA-DR } \\
\text { Double +ve } \\
\text { Double -ve } \\
\text { Single +ve }\end{array}$ & $\begin{array}{c}31 \\
4 \\
22\end{array}$ & $\begin{array}{c}17 \\
1 \\
11\end{array}$ & $\begin{array}{c}73.3 \\
75 \\
63.6\end{array}$ & $\begin{array}{c}11.1 \\
* \\
12.8\end{array}$ & NS & $\begin{array}{c}50 \\
9 \\
38\end{array}$ & $\begin{array}{c}33 \\
6 \\
22\end{array}$ & $\begin{array}{l}87.5 \\
33.3 \\
91.7\end{array}$ & $\begin{array}{l}5.1 \\
1.2 \\
5.4\end{array}$ & NS \\
\hline $\begin{array}{l}\text { FAB subtypes: } \\
\text { M0, MI, M2 } \\
\text { M3 } \\
\text { M4, M5, M7 }\end{array}$ & $\begin{array}{c}35 \\
4 \\
18\end{array}$ & $\begin{array}{c}22 \\
1 \\
6\end{array}$ & $\begin{array}{c}76.5 \\
75 \\
66.7\end{array}$ & $\begin{array}{c}7.6 \\
* \\
*\end{array}$ & - & $\begin{array}{c}56 \\
8 \\
29\end{array}$ & $\begin{array}{c}39 \\
5 \\
17\end{array}$ & $\begin{array}{l}91.1 \\
37.5 \\
79.3\end{array}$ & $\begin{array}{l}5.1 \\
1.2 \\
5.4\end{array}$ & NS \\
\hline $\begin{array}{l}\text { Sex } \\
\text { Males } \\
\text { Female }\end{array}$ & $\begin{array}{l}29 \\
28\end{array}$ & $\begin{array}{l}16 \\
13\end{array}$ & $\begin{array}{c}69 \\
70.4\end{array}$ & $\begin{array}{l}12.1 \\
23.9\end{array}$ & NS & $\begin{array}{l}44 \\
49\end{array}$ & $\begin{array}{l}29 \\
32\end{array}$ & $\begin{array}{l}90.9 \\
87.8\end{array}$ & $\begin{array}{l}6.9 \\
3.6\end{array}$ & NS \\
\hline $\begin{array}{l}\text { Age } \\
\leq 40 \text { years } \\
>40 \text { years }\end{array}$ & $\begin{array}{l}36 \\
21\end{array}$ & $\begin{array}{l}16 \\
13\end{array}$ & $\begin{array}{l}75 \\
60\end{array}$ & $\begin{array}{c}23.9 \\
5.3\end{array}$ & 0.02 & $\begin{array}{l}48 \\
45\end{array}$ & $\begin{array}{l}27 \\
34\end{array}$ & $\begin{array}{l}89.6 \\
86.7\end{array}$ & $\begin{array}{c}13.8 \\
1.3\end{array}$ & 0.002 \\
\hline $\begin{array}{l}\text { TLC } \\
\leq 11 \times 10^{9} / \mathrm{L} \\
>11 \times 10^{9} / \mathrm{L}\end{array}$ & $\begin{array}{l}22 \\
35\end{array}$ & $\begin{array}{l}11 \\
18\end{array}$ & $\begin{array}{l}63.6 \\
76.5\end{array}$ & $\begin{array}{c}12.8 \\
8.8\end{array}$ & NS & $\begin{array}{l}31 \\
62\end{array}$ & $\begin{array}{l}17 \\
44\end{array}$ & $\begin{array}{l}80.5 \\
90.3\end{array}$ & $\begin{array}{l}14.5 \\
2.7\end{array}$ & NS \\
\hline $\begin{array}{l}\text { PB blast: } 50 \% \\
<50 \% \\
\geq 50\end{array}$ & $\begin{array}{l}28 \\
29\end{array}$ & $\begin{array}{l}15 \\
14\end{array}$ & $\begin{array}{l}67.9 \\
71.4\end{array}$ & $\begin{array}{l}11.1 \\
12.8\end{array}$ & NS & $\begin{array}{l}40 \\
53\end{array}$ & $\begin{array}{l}25 \\
36\end{array}$ & $\begin{array}{c}85 \\
88.7\end{array}$ & $\begin{array}{l}7.1 \\
2.5\end{array}$ & NS \\
\hline $\begin{array}{l}\text { BM blast: } 50 \% \\
<50 \% \\
\geq 50\end{array}$ & $\begin{array}{l}17 \\
40\end{array}$ & $\begin{array}{c}9 \\
20\end{array}$ & $\begin{array}{l}52.9 \\
79.5\end{array}$ & $\begin{array}{l}11.1 \\
12.8\end{array}$ & NS & $\begin{array}{l}23 \\
70\end{array}$ & $\begin{array}{l}15 \\
46\end{array}$ & $\begin{array}{l}73.9 \\
91.4\end{array}$ & $\begin{array}{c}8 \\
2.9\end{array}$ & NS \\
\hline $\begin{array}{l}\text { Cytogenetic } \\
\text { analysis: } \\
\text { Favorable } \\
\text { cytogenetics } \\
\text { Intermediate } \\
\text { cytogenetics } \\
\text { Poor cytogenetics }\end{array}$ & $\begin{array}{l}13 \\
9\end{array}$ & $\begin{array}{l}4 \\
6\end{array}$ & $\begin{array}{l}48.6 \\
69.2 \\
33.3\end{array}$ & $\begin{array}{l}\text { II.I } \\
* \\
5.3\end{array}$ & NS & $\begin{array}{l}20 \\
18\end{array}$ & $\begin{array}{l}12 \\
11 \\
14\end{array}$ & $\begin{array}{l}64.7 \\
70 \\
66.7\end{array}$ & $\begin{array}{l}8.4 \\
6.9 \\
1.02\end{array}$ & NS \\
\hline $\begin{array}{l}\text { Flt3 } \\
\text { Wild } \\
\text { Mutant (ITD) }\end{array}$ & $\begin{array}{c}49 \\
4\end{array}$ & $\begin{array}{c}24 \\
3\end{array}$ & $\begin{array}{c}81.6 \\
40\end{array}$ & $\begin{array}{c}12.8 \\
8.8\end{array}$ & NS & $\begin{array}{l}66 \\
14\end{array}$ & $\begin{array}{l}37 \\
12\end{array}$ & $\begin{array}{l}90.9 \\
57.1\end{array}$ & $\begin{array}{l}9.3 \\
1.2\end{array}$ & 0.007 \\
\hline
\end{tabular}

(Continued) 
Table 5 (Continued).

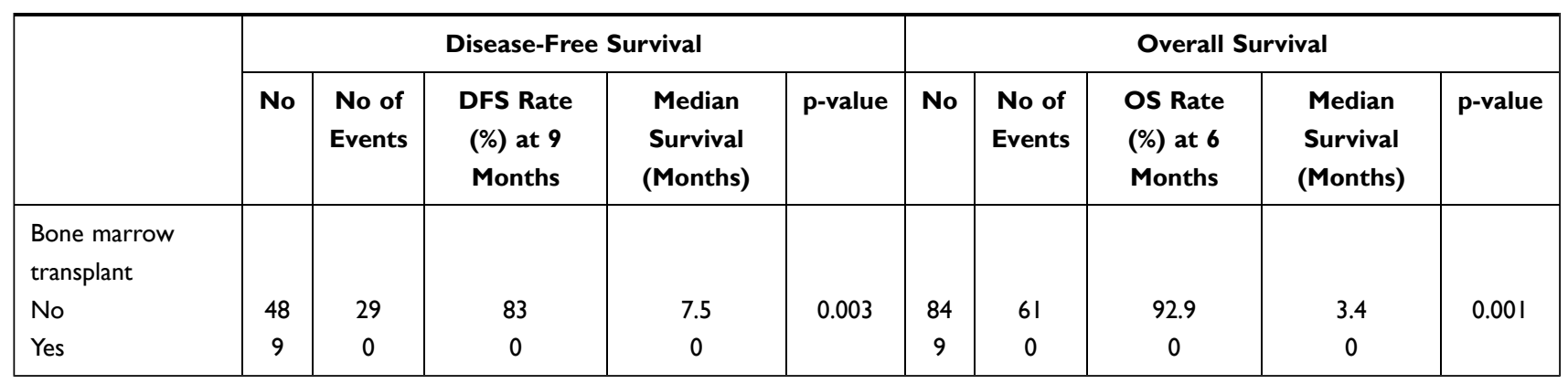

Note: *No median because more than half of the patients were free till the end of the study.

\section{Overall-Survival and Its Relation to Prognostic Factors in AML Patients}

After a median follow-up period of 5.1 months with a range (0.03-24.8) months, $61 \%$ of patients were died [61/100 patients], seven were missing, and 32 still alive till the end of the study. The overall survival rate was $93.5 \%$ (95\% CI; 1.3-9 months). There was a statistically significant association between overall survival and age with a cut-off of 40 years: The survival was better in AML patients less than 40 years $(\mathrm{p}=0.002)$, and also significant association was found between OS and FLT3-ITD: The OS was worse with FLT3-ITD ( $\mathrm{p}=0.007)$. AML patients with bone marrow transplantation had significantly longer overall survival $(\mathrm{p}=0.001)$ [Figure 2], [Table 5].

\section{Disease-Free Survival and Its Relation to Prognostic Factors AML Patients}

Fifty-seven (57) AML patients were achieved complete remission, while 36 patients were resistant to treatment and early died. Cumulative DFS at 9 month for AML patients was $91.1 \%$ (95\% CI; 6.9-17.2 months). There was a statistically significant association between DFS and age with a cut-off of 40 years: the survival was better in AML patients less than 40 years $(p=0.02)$, and also significant association was found between DFS and AML patients without bone marrow transplantation: $46.5 \%$ of patients without bone marrow transplantation still free of the disease till the end of study $(\mathrm{p}=0.003)$ [Figure 2] (Table 5).

\section{Discussion}

AML has been the most common leukemia among adults, but it still has the least survival rate. ${ }^{14}$ Previously, the FAB classification of AML was based on morphological and cytochemical staining properties. Today's WHO classification is based on morphology, immunophenotyping, cytogenetics, and molecular studies that help in better stratification and prognosis. Flow cytometric analysis of acute leukemias provides the fastest and most accurate tool for distinguishing different lineage involvement by the neoplastic process. ${ }^{15}$

HLA-DR has been recognized as a marker that is correlated with early precursor cells and actively metabolizing cells. ${ }^{4}$ The majority of AML cases (about $80 \%$ ) expressed HLA-DR. ${ }^{16}$ CD117 is a tyrosine kinase receptor of the class III subfamily. CD117 expression is typically linked by hematopoietic bone marrow original precursors. It can be recognized through the myeloid cells' lineage until the step of promyelocyte maturation and in the erythroid cells' lineage until the professional erythroblast lineage complete maturation of the stage of the proerythroblast. Generally, CD117 plays a significant role in the viability, proliferation, differentiation, and activation of the hematopoietic progenitor cells $\mathrm{s}^{4.17}$

The results of this study revealed that HLA-DR, CD117, and CD34 expression were assessed in $77.3 \%$, $63 \%$, and $52 \%$, respectively, of all AML patients. The unfavorable genetics FLT3-ITD was found in only 17.1\% of patients. The M3 subtype (APL) accounted for $1.3 \%$ of HLA-DR positive cases compared to $31.8 \%$ of HLA-DR negative patients, with a significant difference between the two values. These results are following the results of previous studies that found HLA-DR-positive APL is very rare. ${ }^{18-20}$ Furthermore, the expression of CD117 was detected in all FAB subtypes, except for M6 and M7, and the expression was highest in M2. CD117 expression is not restricted to any particular, undifferentiated phenotype. $^{4}$ 
Besides, our results showed a significant association between HLA-DR expression and intermediate cytogenetic group (41.9\%) and between HLA-DR negative patients and favorable cytogenetic group (56.2\%). A significant correlation was found between the expression of HLA-DR and CD34 as $72.8 \%$ of HLA-DR negative patients were CD34 negative, and $61.3 \%$ of HLA-DR positive patients were $\mathrm{CD} 34$ positive. When distinguishing non-APL AML from APL cases, the combination of HLADR and CD34 was more helpful in diagnosis than either of these antigens alone.

In our study, Patients with HLA-DR expression showed non-significant worse $\mathrm{CR}$ rates at day $28 \mathrm{com}-$ pared to those without HLA-DR expression $(50 \%$ and $52.4 \%$, respectively), which is similar to the previous study, which found that CR rates were $61 \%$ in HLA-DR positive patients and $73 \%$ in HLA-DR negative patients. ${ }^{21}$ In contrast, another study found that patients' CR rate in the HLA-DR positive group is lower than the negative group, with statistical differences between the two groups. ${ }^{22}$ HLA-DR studies have shown that the risk of recurrence is still high after positive HLA-DR patients reach $\mathrm{CR}^{23}$ Also, Krishnan et al found that HLA-DR had an effective prognosis for elderly AML patients treated with chemotherapy. ${ }^{24}$ These results made a debate about the role of HLA-DR as a prognostic and predictive marker for the response of chemotherapy and indicator to recurrence.

The current study showed that patients with HLA-DR expression had inferior OS compared with those without expression. On the other hand, there was no significant impact of CD117 expression on the clinical outcome of AML patients. Also, FLT3-ITD expression had significantly worse OS.

In this study, we could not find any associations between patient characteristics and the expressions of HLA-DR and CD117 except for platelet counts and lymph node enlargement. The combination of HLA-DR and CD117 expression in our study showed significant associations with AML-FAB subtypes (M0, M1, and M2), CD34 expression, and the intermediate cytogenetic group, which includes AML patients with normal karyotype cytogenetics.

In conclusion, the current study suggested that the expression of CD117 and HLA-DR may be prognostic markers in AML, as the Patients with HLA-DR expression showed significantly higher mean $\mathrm{Hb}$ concentration, higher platelet count associated with AML-FAB subtypes
(M0, M1, and M2), CD34 expression and favorable cytogenetic group. While patients with CD117 expression showed significantly lower platelets count. AML patients with combined HLA-DR and CD117 positive expression are associated with $\mathrm{CD} 34$ expression and intermediate cytogenetic group. However, there was no association between HLA-DR and CD117 expressions, treatment response, and survival rate. Also, remain younger age as a prognostic marker for longer OS. FLT3-ITD had a significant association worse the OS.

\section{Data Sharing Statement}

The data that support the findings of this study are available from the corresponding author upon reasonable request.

\section{Ethics Approval and Consent to Participate}

The study was approved by the Institutional Review Board (IRB) of the NCI, Cairo University, and was conducted using the rules of Helsinki declaration for human studies.

\section{Disclosure}

The authors declare that there are no conflicts of interest in this work.

\section{References}

1. Estey EH. Acute myeloid leukemia: 2012 update on diagnosis, risk stratification, and management. Am J Hematol. 2012;87(1):89-99. doi:10.1002/ajh.22246

2. Elshoura OA, Elkholy RA, Selim AE, et al. Prognostic significance of tetraspanin (CD81) expression in patients with acute myeloid leukemia. Egypt J Haematol. 2018;43(4):151.

3. Boyer T, Guihard S, Roumier C, et al. Tetraspanin CD81 is an adverse prognostic marker in acute myeloid leukemia. Oncotarget. 2016;7 (38):62377. doi:10.18632/oncotarget.11481

4. Döhner H, Estey E, Grimwade D, et al. Diagnosis and management of AML in adults: 2017 ELN recommendations from an international expert panel. Blood. 2017;129(4):424-447. doi:10.1182/blood-201608-733196

5. Sharawat SK, Gupta R, Raina V, et al. Increased coexpression of cKIT and FLT3 receptors on myeloblasts: independent predictor of poor outcome in pediatric acute myeloid leukemia. Cytometry Part B Clin Cytometry. 2013;84(6):390-397.

6. Tarlock K, Eidenschink Brodersen L, Alonzo TA, et al., CD117 expression is associated with cytogenetic and molecular profiles and outcome in pediatric acute myeloid leukemia. Washington, DC: American Society of Hematology; 2019.

7. Seliger B, Kloor M, Ferrone SJO. HLA class II antigen-processing pathway in tumors: molecular defects and clinical relevance. Oncoimmunology. 2017;6(2):e1171447.

8. Arber DA, Orazi A, Hasserjian R, et al. The 2016 revision to the World Health Organization classification of myeloid neoplasms and acute leukemia. Blood. 2016;127(20):2391-2405. doi:10.1182/blood2016-03-643544 
9. Yalniz F, Abou Dalle I, Kantarjian H, et al. Prognostic significance of baseline FLT3-ITD mutant allele level in acute myeloid leukemia treated with intensive chemotherapy with/without sorafenib. $\mathrm{Am}$ J Hematol. 2019;94(9):984-991. doi:10.1002/ajh.25553

10. Gupta M, Mahapatra M, Saxena R. Cytogenetics' impact on the prognosis of acute myeloid leukemia. J Lab Physicians. 2019;11 (2):133. doi:10.4103/JLP.JLP_164_18

11. Abdel Hamid TM, El GammalMM, Eibead GT, et al. Clinical Impact of SNP of P53 Genes Pathway on the Adult AML Patients. Hematology. 2015;20(6):328-335.

12. Cheson BD, Bennett JM, Kopecky KJ, et al. Revised recommendations of the international working group for diagnosis, standardization of response criteria, treatment outcomes, and reporting standards for therapeutic trials in acute myeloid leukemia. J Clin Oncol. 2003;21(24):4642-4649. doi:10.1200/JCO.2003.04.036

13. Ge F, Li B, Gao X, et al. Immuno-phenotypes and prognosis of acute leukemia in elderly patients. Int J Clin Exp Med. 2014;7(10):3714.

14. Marcucci G, Haferlach T, Döhner H. Molecular genetics of adult acute myeloid leukemia: prognostic and therapeutic implications. J Clin Oncol. 2011;29(5):475-486. doi:10.1200/JCO.2010.30.2554

15. Rastogi P, Sachdeva MU. Flow cytometric minimal residual disease analysis in acute leukemia: current status. Indian J Hematol Blood Transfusion. 2020;1-13.

16. Peker D. Navigating through mutations in acute myeloid leukemia. what do we know and what do we do with It? Erciyes Med J. 2018;40 (4):183-187. doi:10.5152/etd.2018.18136
17. Heo S-K, Noh EK, Kim JY, et al. Targeting c-KIT (CD117) by dasatinib and radotinib promotes acute myeloid leukemia cell death. Sci Rep. 2017;7(1):1-12.

18. Mendoza AS, Qing X, Dungo M, et al. HLA-DR antigen-positive acute promyelocytic leukemia. Exp Mol Pathol. 2016;101 (2):197-200.

19. Mosleh M, Mehrpouri M, Ghaffari S, et al. Report of a new six-panel flow cytometry marker for early differential diagnosis of APL from HLA-DR negative Non-APL leukemia. Scandinavian J Clin Lab Invest. 2020;80(2):87-92. doi:10.1080/00365513.2019.1700427

20. Abdul Hamid G, Akrabi M. Aberrant Antigen Expression in Patients with Acute Leukemia. EC Clinical and Medical Case Reports. 2019;2:53-56.

21. Wetzler M, McElwain BK, Stewart CC, et al. HLA-DR antigen-negative acute myeloid leukemia. Leukemia. 2003;17 (4):707-715. doi:10.1038/sj.leu.2402865

22. Xiao S, Zhu HJBR. Correlation study on CD7, CD34, CD56 and HLA-DR expressions and its prognosis among patients with acute myeloid leukemia. Biomedical Research. 2017;28(15). doi:10.1111/ ina. 12430

23. Mori H, Sakai H, Sanada M, et al. Clinical analysis of HLA-DRnegative non-M3 AML. Jpn J Clin Hematol. 2007;48(7):547-553.

24. Krishnan S, Li H, Mourad YR, et al.. Elderly AML patients treated with intensive chemotherapy-developing a prognostic scoring system. a study on 381 patients from British Columbia. American Society of Hematology; 2011.

\section{Publish your work in this journal}

Pharmacogenomics and Personalized Medicine is an international, peer-reviewed, open access journal characterizing the influence of genotype on pharmacology leading to the development of personalized treatment programs and individualized drug selection for improved safety, efficacy and sustainability. This journal is indexed on the American Chemical Society's Chemical Abstracts Service (CAS). The manuscript management system is completely online and includes a very quick and fair peer-review system, which is all easy to use. Visit http://www.dovepress.com/testimonials.php to read real quotes from published authors. 